\title{
Compliance with the Eatwell guide: a case study using supermarket transaction records in Yorkshire and the Humber
}

\section{Abstract}

Introduction: Supermarket transaction data, generated from loyalty cards, offers a novel source of food purchase information. Data are available for large sample sizes, over sustained periods of time, allowing for habitual purchasing patterns to be generated. In the UK, recommended dietary patterns to achieve a healthy diet are pictorially illustrated using the Eatwell Guide. Foods include: Fruit and vegetables; starchy products including potatoes, bread, pasta, rice; dairy or dairy alternatives; proteins such as beans, pulses, fish, eggs and meat; oils and spreads; and advice to limit foods high in salt, fat and sugar. Through mapping of foods purchased to the categories of the Eatwell Guide it is possible to review population performance against these national recommendations.

Materials and methods: All loyalty card transaction records for purchases made in a UK supermarket chain, by residents of Yorkshire and the Humber during 2016 were included in this research. Customers who purchased foods from 7 or 11 Living Cost and Food Survey (LCFS) categories on ten or more occasions throughout the year were included in the sample, as these customers were considered to be purchasing the majority of their foods from the supermarket. All foods purchased were mapped to the Eatwell Guide food groups via the LCFS categories.

Results: Households purchased: $25 \%$ of their total spend on fruits and vegetables, compared with $39 \%$ recommended; $13 \%$ on starchy products compared to $37 \%$ recommended; $23 \%$ of protein rich foods compared with $12 \%$ recommended; $12 \%$ dairy and alternatives compared to $8 \%$; oils and spreads $2 \%$ compared to $1 \%$ recommended; and $25 \%$ foods that should be limited compared to $3 \%$ (recommended, but not pictorially illustrated on the plate).

Discussion: Supermarket transaction data is a novel source of food purchase information which can be used to illustrate dietary behaviours in the UK population. However, it represents foods purchased, not consumed and is at a household level, not individual. Food purchases outside the home are not included. That said, it is arguably an objective measure for dietary assessment. From this study, it is clear to see that food purchases do not match the recommendations. Purchases of high sugar, high fat and high salt snacks constitute a significant proportion of spending, when they should in fact be limited. Protein rich products are also over-represented. Fruit and vegetables and starchy products are under-represented. This insight can benefit both retailers and policy makers for understanding the food purchase behaviours of our society.

\section{Conflict of Interest}

This research was completed by the University of Leeds, using data from Sainsburys. Two co-authors work at the retailer. 